\title{
Thermal Spin Crossover in Fe(II) and Fe(III). Accurate Spin State Energetics at the Solid State
}

\author{
Sergi Vela, ${ }^{a, b^{*}}$ Maria Fumanal, ${ }^{b}$ Jordi Cirera ${ }^{c}$ and Jordi Ribas-Arino ${ }^{d}$ \\ ${ }^{a}$ Laboratory for Computational Molecular Design, Institute of Chemical Sciences and Engineering, EPFL, CH- \\ 1015 Lausanne, Switzerland \\ ${ }^{b}$ Laboratoire de Chimie Quantique, UMR 7111, CNRS-Université de Strasbourg, 4 rue Blaise Pascal, F-67000 \\ Strasbourg, France \\ 'Departament de Química Inorganica i Orgànica and IQTCUB, Universitat de Barcelona, Av. Diagonal 645, \\ 08028, Barcelona, Spain \\ ${ }^{\mathrm{d}}$ Departament de Ciència de Materials i Química Física and IQTCUB, Universitat de Barcelona, Av. Diagonal 645, \\ 08028, Barcelona, Spain \\ *sergi.vela@gmail.com
}

\begin{abstract}
The thermal Spin Crossover (SCO) phenomenon refers to an entropy-driven spin transition in some materials based on $\mathrm{d}_{6}-\mathrm{d}_{9}$ transition metal complexes. While its molecular origin is well known, intricate SCO behaviours are increasingly common, in which the spin transition occurs concomitantly to e.g. phase transformations, solvent absorption/desorption, or order-disorder processes. The computational modelling of such cases is challenging, as it requires accurate spin state energies in the solid state. Density Functional Theory (DFT) is the best framework, but most DFT functionals are unable to balance the spin state energies. While few hybrid functionals perform better, they are still too expensive solid-state minima searches in moderate-size systems. The best alternative is to dress cheap local (LDA) or semi-local (GGA) DFT functionals with a Hubbard-type correction $(\mathrm{DFT}+U)$. However, the parametrization of $U$ is not straightforward due to the lack of reference values, and because ab initio parametrization methods perform poorly. Moreover, SCO complexes undergo notable structural changes upon transition, so intra- and inter-molecular interactions might play an important role in stabilizing either spin state. As a consequence, the $U$ parameter depends strongly on the dispersion correction scheme that is used. In this paper, we parametrize $U$ for nine reported $\mathrm{SCO}$ compounds (five based on $\mathrm{Fe}^{\mathrm{II}}, \mathbf{1 - 5}$ and four based on $\mathrm{Fe}^{\mathrm{III}}, \mathbf{6 - 9}$ ) when using the $\mathrm{D} 3$ and $\mathrm{D} 3-\mathrm{BJ}$ dispersion corrections. We analyze the impact of the dispersion correction treatments on the SCO energetics, structure, and the unit cell dimensions. The average $U$ values are different for each type of metal ion ( $\mathrm{Fe}^{\mathrm{II}} v s$. $\mathrm{Fe}^{\mathrm{III}}$ ), and dispersion correction scheme (D3 vs. D3-BJ) but they all show excellent transferability, with mean absolute errors (MAE) below chemical accuracy (i.e. MAE $<4 \mathrm{~kJ} / \mathrm{mol}$ ). This enables a better description of SCO processes and, more generally, of spin state energetics, in materials containing $\mathrm{Fe}^{\mathrm{II}}$ and $\mathrm{Fe}^{\mathrm{III}}$ ions.
\end{abstract}

\section{Introduction}

Some organometallic complexes based on $\mathrm{d}_{6}-\mathrm{d}_{9}$ transition metal ions undergo a spin-state switch in the presence of an external perturbation. ${ }^{1-8}$ These molecules, called Spin Crossover (SCO), are prototypical class of molecular magnetic switches, ${ }^{9}$ and are regarded as interesting for a range of technological applications. ${ }^{10-13}$ A key property of SCO materials is the temperature at which the spin transition occurs, called transition temperature $\left(T_{1 / 2}\right)$, as it defines their potential applicability in real-life devices operating at mild temperatures. One of the main energetic contributions controlling $T_{1 / 2}$ is the electronic enthalpy difference between the two spin states of the material $\left(\Delta H_{\text {elec }}\right)$. This term control whether a complex is able to undergo thermal SCO, and at which $T_{1 / 2}$, and is also connected to the thermal relaxation temperature $\left(T_{\text {LIESST }}\right)$ when the SCO is triggered by light in the so-called LIESST 
(Light-Induced Excited Spin-State Trapping) effect. ${ }^{14-17}$ In gas-phase conditions, or in the absence of any relevant external influence, $\Delta H_{\text {elec }}$ accounts for the ligand-field splitting $\left(\Delta_{o}\right)$, and intramolecular (e.g. inter-ligand) interactions. In crystals, $\Delta H_{\text {elec }}$ is further affected by the crystal packing effects, which might greatly modify its value. ${ }^{18,19}$ Consequently, it is rather common to find polymorphs or solvatomorphs of the same SCO complex displaying completely different magnetic transitions. ${ }^{20-23}$ Moreover, other solid state phenomena like phase transitions, solvent absorption/desorption or order disorder processes might occur concomitantly to the spin transition, occasionally leading to multiple $\mathrm{SCO}$ pathways, ${ }^{24}$ reverse $\mathrm{SCO},{ }^{25}$ or a spin state blocking along the entire range of temperatures. ${ }^{26,27}$ As a result, the evaluation of $\Delta H_{\text {elec }}$ needs to account for the energetic impact of such processes on the SCO. Indeed, most of the important applications of SCO require the description of its spin state energetics in either the solid-state, in surfaces, or in an interface and, hence, it is crucial to evaluate $\Delta H_{\text {elec }}$ correctly while accounting for the environment.

There are reliable ways to compute $\Delta H_{\text {elec }}$ from electronic structure calculations in gas-, solvent- or crystalline- phase. ${ }^{28-33}$ The best one to treat the latter phase is $\mathrm{DFT}+U$, which implies the dressing of simple DFT functionals with the Hubbard-like term $(U) .^{34-37}$ This scheme has been employed in its GGA $(\mathrm{PBE}+U)$ or LDA $(\mathrm{LDA}+U)$ flavors to study SCO phenomena since the early 2000's, being pioneered by Letard and coworkers, ${ }^{38}$ and Angyan and coworkers. ${ }^{39}$ The main advantage of $\mathrm{DFT}+U$ is its computational cost, enabling minima searches in any complex environment (e.g. solid state). Initially, the DFT $+U$ scheme had two major disadvantages. The treatment of dispersion interactions was restricted to the underperforming LDA or GGA functionals, and the empirical choice of the $U$ parameter undermined its predictive power. The first issue could be tackled $^{40}$ by incorporating cheap dispersioncorrection techniques (such as D2), ${ }^{41}$ whereas the latter was solved by our previous benchmark of the PBE $+U+\mathrm{D} 2$ scheme. ${ }^{19}$ Therein, the adequate value of $U$ able to describe the SCO energetics was determined for a group of seven $\mathrm{Fe}^{\mathrm{II}}$-based compounds. The average value of $U=2.65 \mathrm{eV}$ showed good transferability, with a mean absolute error (MAE) of $4.4 \mathrm{~kJ} / \mathrm{mol}$ when applied to all compounds. Such good accuracy prompted us to tackle a number of puzzling phenomena in the context of thermal SCO, including cooperativity. ${ }^{42}$ The method has been systematically correct in capturing (i) the relative ordering of the LS and HS states (i.e. correct sign of $\Delta H_{\text {elec }}$ ) and (ii) the trends measured experimentally (i.e. correct change of $\Delta H_{\text {elec }}$ ). ${ }^{43-45}$ Moreover, it has provided good quantitative accuracy in comparison to other DFT functionals. However, better dispersion correction schemes have been developed and implemented during this time, and a new benchmark has become necessary. Moreover, the benchmarked $U$ parameter for $\mathrm{Fe}^{\mathrm{II}}$ is likely not adequate to treat $\mathrm{SCO}$ metal complexes based on other metal ions, such as $\mathrm{Fe}^{\mathrm{III}}$, and hence an extension was necessary ( $\mathrm{Fe}^{\mathrm{II}}$ - and $\mathrm{Fe}^{\mathrm{III}}$-based compounds are the largest families of inorganic SCO systems, ${ }^{6,7}$ together with $\mathrm{Co}^{\text {II }} 8$ ).

With this in mind, the motivation of the present paper is, first, to (i) benchmark $U$ under an improved dispersion correction scheme (D3 and D3-BJ) and, second, to (ii) extend the application of the $\mathrm{PBE}+U$ scheme to the family of $\mathrm{Fe}^{\text {III }} \mathrm{SCO}$ compounds. To do so, we have benchmarked the $U$ parameter for five $\mathrm{Fe}^{\mathrm{II}}$ compounds (1-5), and four $\mathrm{Fe}^{\mathrm{III}} \mathrm{SCO}$ compounds (6-9). This benchmark is then complemented with gas-phase computations employing a range of DFT functionals. The results mutually reinforce the use of (i) $\mathrm{PBE}+U$ with the benchmarked $U$ values when in the solid-state, and of B3LYP* (for $\mathrm{Fe}^{\mathrm{II}}$ ) and OLYP (for $\mathrm{Fe}^{\mathrm{III}}$ ) functionals when gas-phase calculations at a higher computational level are feasible. All these schemes achieve chemical accuracy (MAE $\leq 4 \mathrm{~kJ} / \mathrm{mol}$ ). $\mathrm{PBE}+U$, due to is low computational cost, is particularly recommended for the application of modern screening methods and accelerated discovery techniques.

\section{Compounds, methodology, and computational details}

\subsection{Energetics of the SCO transition}

In gradual SCO transitions, the temperature at which the SCO occurs $\left(T_{1 / 2}\right)$ corresponds to the ratio between the enthalpy and entropy differences $\left(\Delta H_{t o t}\right.$ and $\left.\Delta S_{t o t}\right)$ involved in the process. Both terms have two major contributions, electronic and vibrational $\left(\Delta H_{\text {elec }}, \Delta H_{\text {vibr }}, \Delta S_{\text {elec }}\right.$ and $\left.\Delta S_{\text {vibr }}\right)$. The simplest one, $\Delta S_{\text {elec }}$, accounts for the change in electronic degeneracy between the HS and LS states. The vibrational terms $\Delta H_{v i b r}$ and $\Delta S_{v i b r}$ account for the 
Table 1. Experimental thermodynamic data for compounds 1-9 extracted from either DSC measurements or from fitting (see discussion in Section 2.1). Temperature is given in $\mathrm{K}$, enthalpy in $\mathrm{kJ} \cdot \mathrm{mol}^{-1}$ and entropy in $\mathrm{J} \cdot \mathrm{K}^{-1} \cdot \mathrm{mol}^{-1}$.

\begin{tabular}{|l|l|c|c|c|c|}
\hline & \multicolumn{4}{|c|}{ Experimental Estimations } \\
\hline \multicolumn{2}{|c|}{ Compound } & $\boldsymbol{T}_{\mathbf{1 / 2}}$ & $\boldsymbol{\Delta S}_{\boldsymbol{v i b r}}\left(\boldsymbol{T}_{\mathbf{1} / \mathbf{2}}\right)$ & $\boldsymbol{\Delta H}_{\text {tot }}\left(\boldsymbol{T}_{\mathbf{1} / \mathbf{2}}\right)$ & Ref \\
\hline $\mathbf{1}$ & $\mathrm{Fe}^{\mathrm{II}}(\mathrm{phen})_{2}(\mathrm{NCS})_{2}$ & 176 & 35.6 & 8.6 & 46 \\
$\mathbf{2}$ & {$\left[\mathrm{Fe}^{\mathrm{II}}(\mathrm{abpt})_{2}(\mathrm{NCS})_{2}\right]$} & 180 & 19.1 & 5.8 & 2 \\
$\mathbf{3}$ & {$\left[\mathrm{Fe}^{\mathrm{II}}(\mathrm{abpt})_{2}(\mathrm{NCSe})_{2}\right]$} & 224 & 24.6 & 8.6 & 2 \\
$\mathbf{4}$ & $\mathrm{Fe}^{\mathrm{II}}\left(\mathrm{HB}(\mathrm{pz})_{3}\right)_{2}$ & 2350 & 36.6 & 17.5 & 47 \\
$\mathbf{5}$ & {$\left[\mathrm{Fe}^{\mathrm{III}}(1-\mathrm{bpp})\right]\left[\mathrm{BF}_{4}\right]_{2}$} & 259 & 52.8 & 17.2 & 26 \\
\hline $\mathbf{6}$ & {$\left[\mathrm{Fe}^{\mathrm{III}}\left(\mathrm{L}^{1}\right)(\mathrm{NCS})\right]$} & 114 & 8.3 & 1.6 & 48 \\
$\mathbf{7}$ & {$\left[\mathrm{Fe}^{\mathrm{III}}\left(\mathrm{L}^{\mathrm{Cl}}\right)(\mathrm{NCS})\right]$} & 280 & 14.8 & 5.8 & 48 \\
$\mathbf{8}$ & {$\left[\mathrm{Fe}^{\mathrm{III}}\left(\mathrm{L}^{\mathrm{Br}}\right)(\mathrm{NCSe})\right]$} & 317 & 13.7 & 6.2 & 49 \\
$\mathbf{9}$ & {$\left[\mathrm{Fe}^{\mathrm{III}}(\mathrm{qsal}-\mathrm{I})_{2}\right] \mathrm{Otf} \cdot \mathrm{MeOH}$} & 232 & 5.6 & 23.8 & 50
\end{tabular}

change in the vibrational levels of the molecule, mostly due to the occupation of the antibonding $e_{g}$ orbitals and the concomitant expansion of the coordination sphere upon SCO. In a crystal, these terms also account for the change of lattice phonons. $\Delta H_{v i b r}$ can be safely modelled using the Harmonic-Oscillator (HO) model, whereas $\Delta S_{v i b r}$ is better treated combining the $\mathrm{HO}$ and Free-Rotor (FR) models. ${ }^{51}$ Both models use the vibrational normal modes $\left(v_{i}\right)$, whose computational evaluation remains a challenge. The computational cost of accurately evaluating $v_{i}$ in molecular crystals is large, so calculations usually restrict to isolated molecules. As a result, the evaluation of $\Delta H_{v i b r}$ and $\Delta S_{v i b r}$ does not incorporate the effect of lattice vibrations, intermolecular interactions and anharmonicity, which might occasionally have an impact on $v_{i}$ and, thus, on the vibrational contributions. ${ }^{52,53}$

\subsection{List of compounds.}

Our main criteria to choose the studied compounds has been: (i) available crystal structures, without significant disorder, (ii) complete, single-step spin transition and, when possible, (iii) calorimetry measurements offering an experimental estimate of $\Delta H_{t o t}$ and $\Delta S_{t o t}$, which facilitate the assignation of a reference $\Delta H_{\text {elec }}$ value (i.e. $\Delta H_{\text {elec }}^{\text {ref }}$ ) from which we can benchmark our methodology. Moreover, we have favored gradual and, specially, non-hysteretic SCO. The reason is that systems in which cooperativity has a complex energetic fingerprint, $T_{1 / 2}$ is no longer defined as the ratio between the enthalpy and entropy differences. ${ }^{42}$ The group of $\mathrm{Fe}^{\mathrm{II}}$ compounds includes (i) the ubiquitous $\mathrm{Fe}(\text { phen })_{2}(\mathrm{NCS})_{2}(\mathbf{1})$ of Sorai and Seki, ${ }^{54}$ in the crystal structure reported by Gallois, ${ }^{46}$ (ii) the $\mathrm{X}=\mathrm{S}$ (2) and $\mathrm{X}=\mathrm{Se}(3)$ variations of $\left[\mathrm{Fe}(\mathrm{abpt})_{2}(\mathrm{NCX})_{2}\right]$ reported by Moliner, ${ }^{55}$ (iii) the $\mathrm{Fe}\left[\mathrm{HB}(\mathrm{pz})_{3}\right]_{2}$ (4) of Bousseksou ${ }^{47}$ and, finally, (iv) $[\mathrm{Fe}(1-\mathrm{bpp})]\left[\mathrm{BF}_{4}\right]_{2}$ (5) of Halcrow. ${ }^{26}$ This set of compounds is almost the same we studied previously in ref. ${ }^{19}$. An exception are compounds $\left[\mathrm{Fe}\left(\mathrm{HB}(\mathrm{pz})_{3}\right]_{2}\right.$ and $\mathrm{Fe}\left[\mathrm{H}_{2} \mathrm{~B}(\mathrm{pz})_{2}\right]_{2}$ (bipy) of our original benchmark, which have not been included in this study. For the former compound, the reason is that its experimental $\Delta H_{\text {tot }}$ and $\Delta S_{\text {tot }}$ values refer to the first jump (from LS to 1:1 HS:LS) of its two-step transition, whereas our calculations assumed the full LS-to-HS transformation. This was already mentioned in our original paper, but not properly tackled, as it should have never been used as a good reference value. For the latter, the available estimations of $\Delta H_{t o t}$ and $\Delta S_{\text {tot }}$ were obtained from a fitting of the $\chi \mathrm{T}$ curve with the SlichterDrickammer model..$^{56}$ The fitted value of $\Delta S_{\text {tot }}$ (81.9 $\mathrm{J} / \mathrm{K} \cdot \mathrm{mol}$ ) is $25 \%$ larger than the largest value in our library of $\mathrm{Fe}^{\mathrm{II}}$ compounds so we did not considered it reliable (see Table 1) and, hence, we disregarded this compound. The list of $\mathrm{Fe}^{\mathrm{III}}$ compounds has been much more difficult to establish. A comparatively-small amount of works in the literature report DSC measurements, and only few are free from disorder, symmetry breaking, solvent evaporation or hysteresis. Our final list includes three compounds synthesized by Renz and coworkers: $\left[\mathrm{Fe}^{\mathrm{III}}\left(\mathrm{L}^{1}\right)(\mathrm{NCS})\right] \quad(\mathbf{6})$, $\left[\mathrm{Fe}^{\mathrm{III}}\left(\mathrm{L}^{\mathrm{Cl}}\right)(\mathrm{NCS})\right](\mathbf{7})$ and $\left[\mathrm{Fe}^{\mathrm{IIII}}\left(\mathrm{L}^{\mathrm{Br}}\right)(\mathrm{NCSe})\right](\mathbf{8}),{ }^{48,}, 49$ in which the thermodynamic quantities were extracted using an Ising-like model developed by Boča and coworkers, which provided very robust values that are in line with the existing literature. ${ }^{57}$ The last system is $\left[\mathrm{Fe}(\mathrm{qsal}-\mathrm{I})_{2}\right] \mathrm{Otf} \cdot \mathrm{MeOH}(\mathbf{9})$ reported by Harding, ${ }^{50}$ in 
Table 2. Calculated thermodynamic data for compounds 1-9, and benchmarked U values under the $D 3$ and $D 3-B J$ schemes. Temperature is given in $\mathrm{K}$, enthalpy in $\mathrm{kJ} \cdot \mathrm{mol}^{-1}$, entropy in $\mathrm{J} \cdot \mathrm{K}^{-1} \cdot \mathrm{mol}^{-1}$, and the $U$ values in $\mathrm{eV}$. The thermodynamic data is given per molecule.

\begin{tabular}{|c|c|c|c|c|c|}
\hline & $\Delta H_{t o t}$ & $\Delta H_{v i b}\left(T_{1 / 2}\right)$ & $\Delta H_{e l e c}^{r e f}$ & $U-D 3$ & $U-D 3-B J$ \\
\hline 1 & 8.6 & -6.4 & 15.0 & 2.05 & 2.12 \\
\hline 2 & 5.8 & -7.4 & 13.2 & 2.28 & 2.32 \\
\hline 3 & 8.6 & -6.4 & 14.5 & 2.33 & 2.38 \\
\hline 4 & 17.5 & -3.9 & 21.4 & 2.44 & 2.58 \\
\hline 5 & 17.2 & -2.0 & 19.1 & 2.33 & 2.35 \\
\hline \multicolumn{4}{|r|}{ Avg. } & 2.29 & 2.35 \\
\hline 6 & 1.6 & -8.7 & 10.3 & 2.15 & 2.19 \\
\hline 7 & 5.8 & -5.9 & 11.7 & 2.49 & 2.48 \\
\hline 8 & 6.2 & -5.7 & 11.9 & 2.58 & 2.56 \\
\hline 9 & 23.8 & -5.3 & 10.9 & 2.56 & 2.43 \\
\hline \multicolumn{4}{|r|}{ Avg. } & 2.44 & 2.39 \\
\hline
\end{tabular}

which the thermodynamic parameters were extracted from DSC measurements (see Table 1). The structure of 1-9 is represented in Fig. S1.

\subsection{D2 vs. D3 vs. D3-BJ dispersion corrections.}

The main difference between the D2 and D3 corrections is that, in the former, the $C_{6}$ coefficients are based exclusively on the atom type, whereas D3 takes into account also their chemical environment through a coordination number. Accordingly, the $C_{6}$ and $C_{8}$ coefficients are also different. Finally, each scheme uses a different damping function aimed at correcting the energetic contribution at very close ranges (Fermi function in D2, Chai and Head-Gordon ${ }^{58}$ in D3, and Becke and Johnson ${ }^{59-61}$ in D3-BJ). Further information about dispersion corrections in general, and the D2 and D3 in particular, can be found in the literature. ${ }^{62,63}$

\subsection{Estimation of reference values and U benchmark.}

Following the working strategy we used in our previous benchmark of the $\mathrm{PBE}+U+\mathrm{D} 2$ scheme, ${ }^{19}$ we have estimated $\Delta H_{v i b}$ and $\Delta H_{\text {elec }}^{\text {ref }}$ for compounds 1-9 (see Table 2). The strategy consists in extracting $\Delta H_{\text {elec }}^{\text {ref }}$ from the experimental (from DSC or fitted) value of $\Delta H_{\text {tot }}$ and a calculated value of $\Delta H_{v i b} \cdot \Delta H_{v i b}$ is computed from slightly-modified vibrational normal modes $v_{i}$ of the isolated molecule: the eight lowerfrequency $v_{i}$ of the LS and HS minima are adjusted to reproduce the $\Delta S_{v i b}$ value that results from subtracting $\Delta S_{\text {elec }}$ from the experimental $\Delta S_{\text {tot }}$. Given that $\Delta H_{v i b}$ is mostly affected by the high-frequency $v_{i}$, this adjustment does not have a significant impact on $\Delta H_{v i b}$ and, hence, on $\Delta H_{\text {elec }}^{\text {ref }}$. Therefore, the resulting $\Delta H_{\text {elec }}^{\text {ref }}$ value is our best estimate of the adiabatic energy difference between the HS and LS states of compounds 1-9 in the solid state (see Table 2).

Once the reference $\Delta H_{\text {elec }}^{\text {ref }}$ values have been estimated, the Hubbard-like $U$ parameter under the $\mathrm{PBE}+U+\mathrm{D} 3$ and $\mathrm{PBE}+U+\mathrm{D} 3-\mathrm{BJ}$ schemes is parametrized to reproduce these values. We start by performing variable-cell geometry optimizations to obtain the minima of the HT and LT phases of each compound. Then, at these fixed structures, we perform single-point energy evaluations at different values of $U$ ranging from 1.6 and $3.0 \mathrm{eV}$. Within this range, the evolution of $\Delta H_{\text {elec }}$ is mostly linear, so the exact $U$ value reproducing $\Delta H_{e l e c}^{\text {ref }}$ is easily interpolated (see Figure S2). Notice that, in this benchmark, our philosophy is to employ the same $U$ value for both the LS and HS states of the material. Also, our method is empirical in the sense that the $U$ values are benchmarked to reproduce estimated $\Delta H_{\text {elec }}$ values. This strategy is typically discouraged in favor of ab-initio benchmarking methods that would result in different $U$ values for each of the LS and HS minima. ${ }^{37,64}$ However, this method does not seem suitable to describe the spin state energetics of SCO compounds, since it leads to a $\Delta H_{\text {elec }}$ value of $-175 \mathrm{~kJ} / \mathrm{mol}$ for compound $\mathbf{1}$, with selfconsistent $U$ values of 6.17 and $4.09 \mathrm{eV}$ for its $\mathrm{LS}$ and HS states, respectively (see Section $\mathrm{S} 2$ ). The resulting 
self-consistent $U$ values are, thus, overestimated, as already pointed out in the literature for Heme $\mathrm{Fe}^{\mathrm{II}}$-based molecules. ${ }^{65}$

\subsection{Computational Details}

$P B E+U$ calculations in the solid state. Optimization of the HS and LS crystals for all compounds was performed with Quantum Espresso at $\mathrm{PBE}+U$ level ( $U=2.65$ for $\mathrm{Fe}^{\mathrm{II}}$ and $U=2.0$ for $\mathrm{Fe}^{\mathrm{III}}$ complexes) complemented with the D2, D3 and D3-BJ dispersion corrections. We have used Vanderbilt ultrasoft pseudopotentials. ${ }^{66}$ All computations have been performed at the $\Gamma$-point of the Brillouin zone. The minimum energy structure has been obtained by performing successive variable-cell geometry relaxations, in which the lattice parameters as well as the atomic positions are optimized simultaneously until the atomic forces are smaller than $1.0 \cdot 10^{-5}$ atomic units. In these calculations, the number of plane waves has been kept constant at a kinetic energy cutoff of 70 Ry for the wavefunction (ecutwfc) and of $560 \mathrm{Ry}$ for the charge density (ecutrho) throughout the variable-cell relaxations. A final SCF calculation has been done at the final optimized structure with kinetic energy cutoffs of 35 and 280 Ry for ecutwfc and ecutrho, respectively. The spin state of the iron atoms is defined in the initial guess, and maintained along the optimization. We must note here that the LS crystal structure for compounds 2 and $\mathbf{3}$ has not been reported experimentally. In those cases, and given that the spin transition neither implies a change in the symmetry of the crystal, nor in the orientation of the molecules, the LS minima have been obtained starting from the HS crystals.

$P B E+U$ calculation in the gas phase. To calculate the energy of the isolated molecules, the coordinates of a SCO molecule, whose typical size is ca. $35 \mathrm{Bohr}^{3}$, have been excised from the optimized unit cell and introduced on a cubic cell of $60 \mathrm{Bohr}^{3}$. This fact, together with the application of the Makov-Payne correction within QE, ${ }^{67}$ ensures that the molecules are isolated from their virtual counterparts. In these calculations, the geometry has not been optimized in order to preserve the structure found in the variable-cell geometry optimizations. If non-identical SCO units have been found on the optimized crystal, this process has been done for each of those, and the results correspond to an average. In Section 3.3, we have tested several exchange correlation functionals at describing the correct spin-state energy differences, including OLYP, OPBE, B3LYP, B3LYP*, TPSSh, M06L and SCAN. All single point computations have been performed with the quantum chemistry package QChem 5.0. ${ }^{68}$ The def2-TZVP ${ }^{69,70}$ basis set was used on all atoms, and a convergence criterion on the energy of $10^{-8}$ was employed for all calculations. Dispersion correction effects were included via Grimme's D3 and D3-BJ schemes. ${ }^{71}$

\section{Results and Discussion}

\subsection{Estimation of $\Delta H_{\text {elec }}^{\text {ref }}$ and U for 1-9}

The protocol described in Section 2.3 to extract the reference $\Delta H_{\text {elec }}^{\text {ref }}$ values has been applied to compounds 1-9 (see Table 1). Those of the Fe ${ }^{\mathrm{II}}$ subset correlate very well with the experimental $T_{1 / 2}$. In turn, the Fe ${ }^{\mathrm{III}}$ subset shows very similar $\Delta H_{\text {elec }}^{\text {ref }}$ values despite their $T_{1 / 2}$ ranges from 114 to $344 \mathrm{~K}$. Using the D3 correction, the average $U$ values of the $\mathrm{Fe}^{\mathrm{II}}$ and $\mathrm{Fe}^{\mathrm{III}}$ complexes are quite different (2.29 vs. $2.44 \mathrm{eV})$, while the D3-BJ correction leads to much more similar values of $U(2.35$ vs. $2.39 \mathrm{eV}$ ). For Fe ${ }^{\mathrm{II}}$ compounds, both D3 and D3-BJ values are significantly lower than the one we benchmarked with $\mathrm{PBE}+U+\mathrm{D} 2$ (i.e. $2.65 \mathrm{eV}$ ). As we will discuss later, the reason is that the D2 correction has a much larger contribution to $\Delta H_{\text {elec }}$ than D3 and D3-BJ, especially when it comes to the description of the intramolecular interactions within the SCO molecules (see Figure 1). Overall, this results in a net overestabilization of the LS state when using D2 (see Table 3), that needs to be corrected through a larger $U$ parameter.

\subsection{Role of Intermolecular Interactions}

Using the benchmarked $U$ value for each compound and each scheme (D3 and D3-BJ, see Table 2), we have computed $\Delta H_{\text {elec }}$ for isolated molecules of 1-9 $\left(\Delta H_{\text {elec }}^{\text {iso }}\right)$ excised (i.e. without further optimization) from the respective solid-state minima. The difference between $\Delta H_{e l e c}^{i s o}$ and $\Delta H_{e l e c}^{\text {ref }}$ is the energetic influence of the crystal packing to the SCO transition through intermolecular interactions of any kind (dispersion, induction, electrostatic, steric). The crystal-packing effects $(\mathrm{CPE})$ reported herein range from -14.1 to +8.0 


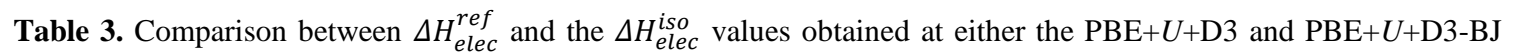
minima computed in this work, or the $\mathrm{PBE}+U+\mathrm{D} 2$ minima in reference 19 , using the parametrized $U$ values, and the D2, D3 and D3-BJ corrections, respectively. The difference between $\Delta H_{\text {elec }}^{\text {ref }}$ and $\Delta H_{\text {elec }}^{\text {iso }}$ are crystal packing effects (CPE), which account for any kind of intermolecular interaction (electrostatic, dispersion, steric hindrance, etc.). All energy contributions are given per molecule.

\begin{tabular}{|c|c|cc|cc|cc|}
\hline & & \multicolumn{2}{|c|}{ D2 minima $\left(\right.$ ref. $\left.^{19}\right)$} & \multicolumn{2}{c|}{ D3 minima (this work) } & \multicolumn{2}{c|}{ D3-BJ minima (this work) } \\
\hline & $\boldsymbol{\Delta H}_{\text {elec }}^{\text {ref }}$ & $\boldsymbol{\Delta H}_{\text {elec }}^{\text {iso }}$ & $\boldsymbol{C P E}$ & $\boldsymbol{\Delta H}_{\boldsymbol{e l e c}}^{\boldsymbol{i s o}}$ & $\boldsymbol{C P E}$ & $\boldsymbol{\Delta} \boldsymbol{H}_{\text {elec }}^{\text {iso }}$ & $\boldsymbol{C P E}$ \\
\hline $\mathbf{1}$ & 15.0 & 6.9 & 8.1 & 9.4 & 5.6 & 9.0 & 6.0 \\
$\mathbf{2}$ & 13.2 & 5.9 & 7.3 & 16.6 & -3.4 & 15.4 & -2.2 \\
$\mathbf{3}$ & 14.5 & 7.6 & 6.9 & 21.3 & -6.8 & 17.8 & -3.3 \\
$\mathbf{4}$ & 21.4 & 22.9 & -1.5 & 35.3 & -14.1 & 35.0 & -13.6 \\
$\mathbf{5}$ & 19.1 & 15.9 & 3.2 & 20.6 & -1.5 & 20.6 & -1.5 \\
\hline $\mathbf{6}$ & 10.3 & - & - & 2.3 & 8.0 & 1.8 & 8.5 \\
$\mathbf{7}$ & 11.7 & - & - & 5.2 & 6.5 & 5.1 & 6.6 \\
$\mathbf{8}$ & 11.9 & - & - & 6.1 & 5.8 & 6.9 & 5.0 \\
$\mathbf{9}$ & 10.9 & - & - & 10.9 & 0.0 & 11.6 & -0.7 \\
\hline
\end{tabular}

$\mathrm{kJ} / \mathrm{mol}$ (with D3), and might contribute to the stabilization of either the HS or LS state. Overall, D2 and D3-based schemes describe much differently the landscape of interactions. Those associated with the $\mathrm{Fe}^{\mathrm{II}}$ systems (i.e. 1-5) are significantly different than the ones we reported in ref. 19 when using the D2 scheme (see Table 3). For most compounds, D2 describes that intermolecular interactions lead to an overall stabilization of the LS state (CPE entry is positive in Table 3), whereas D3 corrections describe the contrary ( $C P E$ entry is smaller or negative). One possible reason is that D3 and D3-BJ corrections leads to larger unit cells than the D2 in both spin states (although the unitcell expansion upon $\mathrm{SCO}$ is similar, see $\Delta V$ in Table $3)$.Indeed, in some cases the volume of the predicted minima is even larger than the reported X-ray structures, which suggests that D3 and D3-BJ might unrealistically overestimate the unit cell volume. This is consistent with other studies showing that dispersion corrected GGAs like PBE-D3, used in periodic plane-wave DFT computations, systematically overestimate the molecule volume by roughly $2 \%$. The reason might the inclusion of the three-body term, which is repulsive for all solids, ${ }^{72,73}$ and of the damping functions used in D3 and D3-BJ. ${ }^{63}$

To get better insight, we now trace the D2, D3 and D3BJ energy contribution to $\Delta H_{\text {elec }}$ at the same set of PBE $+U+\mathrm{D} 3$ solid-state minima (see Figure 1). These comparison enables us to identify to what extend the contribution to $\Delta H_{\text {elec }}$ shown in Table 3 stems from the construction of the different dispersion correction schemes (see section 2.3). Additionally, we have also computed and displayed its intra-molecular component (dashed bars in Figure 1). The difference between filled and dashed bars corresponds to the inter-molecular component.

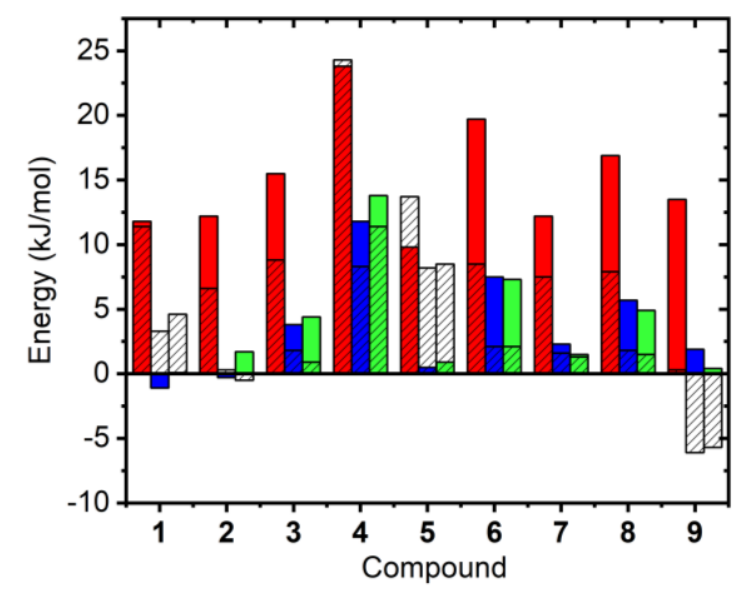

Figure 1. Energy contribution to $\Delta H_{\text {elec }}$ arising from dispersion interactions as described by the D2 (red), D3 (blue) and D3-BJ (green) dispersion correction schemes at the same solid-state minima computed with $\mathrm{PBE}+U+\mathrm{D} 3$. Positive (negative) values indicate that the LS (HS) state is favored. Intra-molecular contributions are shown as dashed patterns. All energy contributions are given per molecule.

Similar to what we mentioned earlier, the overall picture suggests that the use of D2 leads to a much larger influence of dispersion interactions to the SCO energetics, favoring the LS state (all red bars show positive values in Figure 1). In turn, D3 and D3-BJ greatly diminish their impact up to the point that, for most systems, it becomes negligible. 
D3

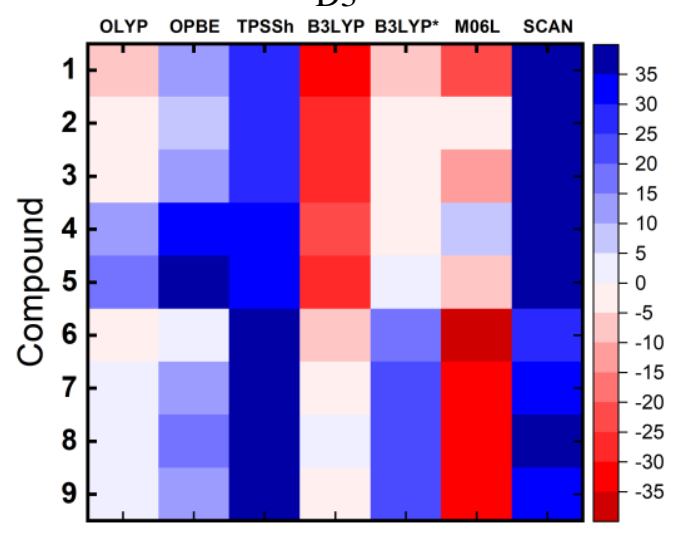

D3-BJ

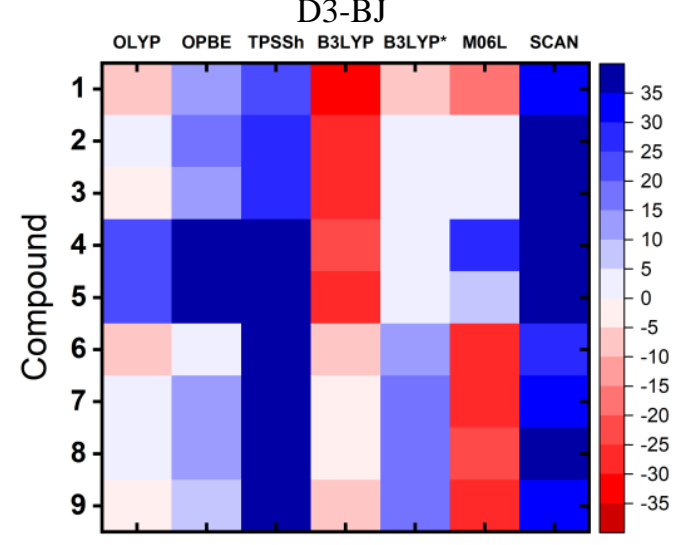

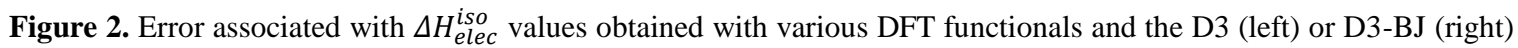
dispersion correction scheme. The reference is the $\Delta H_{\text {elec }}^{\text {iso }}$ value obtained with $\mathrm{PBE}+U$ using the benchmarked $U$ (different for each compound, see Table 2) at isolated molecules of 1-9 excised from the PBE+ $U+\mathrm{D} 3$ (left) and PBE $+U+\mathrm{D} 3-\mathrm{BJ}$ (right) solid-state minima. Blue (red) indicates that the LS (HS) is too stable according to the functional. See also Tables S4-S6.

Further comparison between compounds would not meaningful due to the different molecular and unit cell composition. A much broader analysis should be carried out to analyze this issue in detail ${ }^{45}$ which is out of the scope of this work.

\subsection{Gas phase PBE+U vs. Other DFT Functionals}

The PBE $+U$ results in periodic boundary conditions, are now complemented with gas-phase calculations using a range of DFT functionals. These are carried out at the molecular geometries of 1-9 excised from the solid-state minima without further optimization. The main motivation of these calculations is to investigate the performance of DFT functionals when they are confronted to sound reference values $\left(\Delta H_{\text {elec }}^{\text {iso }}\right)$. We have chosen the exchange/correlation functionals OLYP, OPBE, TPSSh, B3LYP, B3LYP*, M06L and SCAN, (complemented with the D3 and D3-BJ corrections), which have shown different degrees of success in calculating spin-state energies in SCO systems in the past. $^{28}$ The results are summarized on Figure 2, and numerical data can be found in Tables S4 and S5. The functionals TPSSh and SCAN correctly reproduce the ground state, but only as a result of a very large overestabilization of the LS state. Therefore, they do not describe compounds 1-9 as SCO-capable. B3LYP, OPBE and M06L perform slightly better, but the former two often fail at assigning the correct ground state. On the bright side, B3LYP* provides very similar spin-state energies to $\mathrm{PBE}+U$, and OLYP achieves the best mean average error (MAE) for the whole dataset (MAE=6.9 $\mathrm{kJ} / \mathrm{mol}$ ). Further insight can be achieved doing a separate analysis of the results for $\mathrm{Fe}^{\mathrm{II}}$ and $\mathrm{Fe}^{\mathrm{III}}$ compounds. If we focus on the $\mathrm{Fe}^{\mathrm{II}}$ dataset, the MAE associated with B3LYP* is only $0.79 \mathrm{~kJ} / \mathrm{mol}$, which is quite remarkable. Indeed, B3LYP* was reparametrized specifically to model spin-state energies in $\mathrm{Fe}^{\mathrm{II}} \mathrm{SCO}$ systems, ${ }^{74,75}$ and such tiny error does serve to validate the benchmarked $U$ values for 1-5. For the Fe ${ }^{\mathrm{III}}$ systems, the $\mathrm{PBE}+\mathrm{D} 3$ results are in very good agreement with the ones obtained with the B3LYP and the OPBE functionals (MAE $=0.84$ and $2.85 \mathrm{~kJ} / \mathrm{mol}$, respectively). The latter has already demonstrated its accuracy towards SCO systems, ${ }^{76,77}$ and in particular towards $\mathrm{d}^{5}$ SCO molecules before, ${ }^{78}$ which yet again reinforces the benchmarked $U$ values for 6-9. In turn, the good performance of B3LYP is somehow surprising, although the higher ionic character (and hence larger $\Delta_{o}$ ) of $\mathrm{Fe}^{\mathrm{III}}$, with respect to $\mathrm{Fe}^{\mathrm{II}}$, should explain the need for a larger HF-exchange percentage (B3LYP $20 \%$ vs. B3LYP* 15\%).

\subsection{Transferability}

In section 3.1 we have obtained the $U$ values that reproduce $\Delta H_{\text {elec }}^{\text {ref }}$ for each system. Eventually, when studying new SCO systems, one would like to skip such benchmark and instead use an approximate value that guarantees the smallest possible error. Logically, one 
would favor using the average $U$ values proposed in section 3.1 for $\mathrm{PBE}+U+\mathrm{D} 3(2.29$ and $2.44 \mathrm{eV})$ and PBE $+U+\mathrm{D} 3-\mathrm{BJ}(2.35$ and $2.39 \mathrm{eV})$. To have an estimation of the error that such approach would entail, single-point computations are performed on very same solid state minima (the geometry is not affected by $U$ ) using these $U$ values. The resulting $\Delta H_{\text {elec }}$ are then compared to $\Delta H_{\text {elec }}^{r e f}$ and the difference is the error associated with the use of those average values (instead of the best parametrization for each compound). The mean absolute error (MAE) is of $4.5\left(\mathrm{Fe}^{\mathrm{II}}\right)$ and 2.8 $\mathrm{kJ} / \mathrm{mol}\left(\mathrm{Fe}^{\mathrm{III}}\right)$ for the PBE+ $U+\mathrm{D} 3$ minima, and of 4.4 $\left(\mathrm{Fe}^{\mathrm{II}}\right)$ and $2.3 \mathrm{~kJ} / \mathrm{mol}\left(\mathrm{Fe}^{\mathrm{III}}\right)$ with $\mathrm{PBE}+U+\mathrm{D} 3-\mathrm{BJ}$ (see Table S7). As expected, the error is much larger in systems whose benchmarked $U$ is further away from the average $U(\mathbf{1}, \mathbf{4}$ and $\mathbf{6})$. In the case of $\mathbf{1}$, one possible reason is the reported importance of anharmonic effects in its SCO transition. ${ }^{52}$ Finally, we notice that our previous parametrization of $\mathrm{PBE}+U+\mathrm{D} 2$, with $U=2.65$ $\mathrm{eV}$, yielded a MAE of 5.1 for compounds 1-5, so we can infer that the new parametrization does improve the transferability of the method.

\section{Conclusions}

We have parametrized the $U$ values that must be employed to describe the energetics of the thermal SCO transition of five $\mathrm{Fe}^{\mathrm{II}}(\mathbf{1 - 5})$ and four $\mathrm{Fe}^{\mathrm{III}}$ compounds (69), under the $\mathrm{PBE}+U+\mathrm{D} 3$ and $\mathrm{PBE}+U+\mathrm{D} 3-\mathrm{BJ}$ methods. The average $U$ values that result: $2.29\left(\mathrm{Fe}^{\mathrm{II}}\right)$ and $2.44 \mathrm{eV}$ $\left(\mathrm{Fe}^{\mathrm{III}}\right)$ for the former method, and $2.35\left(\mathrm{Fe}^{\mathrm{II}}\right)$ and 2.39 $\mathrm{eV}\left(\mathrm{Fe}^{\mathrm{III}}\right)$ for the latter, can be used reliably to describe other $\mathrm{Fe}^{\mathrm{II}}$ and $\mathrm{Fe}^{\mathrm{III}} \mathrm{SCO}$ systems with chemical accuracy. Moreover, and due to its local nature, different $U$ values can be applied to different metal ions in the same computation, which enables the accurate description of mixed $\mathrm{Fe}^{\mathrm{II}}-\mathrm{Fe}^{\mathrm{III}}$ systems (i.e. double $\mathrm{SCO}$ salts $\left.{ }^{79}\right)$. Concerning the dispersion corrections, we noticed that the use of D3-based dispersion correction schemes leads to a different landscape of contributions to $\Delta H_{\text {elec }}$ arising from dispersion interactions than with D2. The latter correction seems to overestimate the importance of dispersion interactions to the SCO transition, with a net stabilization of the LS state that must be compensated by larger $U$ values. Finally, we compared the performance of other DFT functionals at describing the spin state energetics of 1-9 in gas-phase, and the geometry of the solid state minima. Both using $\mathrm{D} 3$ and D3-BJ dispersion corrections, we found that $\mathrm{Fe}^{\mathrm{II}}$ and $\mathrm{Fe}^{\mathrm{III}}$ complexes are better described using B3LYP* and OLYP, respectively (MAE of 3.5 and $2.9 \mathrm{~kJ} / \mathrm{mol}$ respectively for $\mathrm{D} 3$ ). This finding complements was is known from the existing literature on the subject, and serves to further validate the benchmark of the $\mathrm{PBE}+U+\mathrm{D} 3$ and $\mathrm{PBE}+U+\mathrm{D} 3-\mathrm{BJ}$ schemes.

\section{Acknowledgements}

S.V. and M.F. are thankful to the ANR and the LabEx programs for their postdoctoral grants (ANR-10-LABX-0026_CSC), and to the regional HPC center in Strasbourg for the allocation of computer time. S.V. acknowledges discussions with Prof. D. J. Harding.

\author{
Abbreviations \\ Phen=1,10-Phenanthroline \\ Abpt=4-Amino-3,5-bis(pyridin-2-yl)-1,2,4-triazole \\ $\mathrm{Pz}=$ Pyrazine \\ 1-bpp=2,6-Di(pyrazol-1-yl)pyridine \\ $\mathrm{H}_{2} \mathrm{~L}^{1}=1-[3-[2-(2-$ hydroxynaphthalen $-1-\mathrm{yl})$ methylenaminoethylam \\ ino]propylimino]methyl)naphthalene-2-ol. \\ $\mathrm{H}_{2} \mathrm{~L}^{\mathrm{Cl}}=\mathrm{N}, \mathrm{N}$ '-bis(1-hydroxy-4-chloro-2-benzyliden)-1,6-diamino \\ -4-azahexane \\ $\mathrm{H}_{2} \mathrm{~L}^{\mathrm{Br}}=\mathrm{N}, \mathrm{N}$ '- bis(1-hydroxy-4-bromo-2-benzyliden $)-1,6$-diamino \\ -4-azahexane \\ qsal-I= quinolylsalicylaldiminate \\ Otf $=$ Triflate
}

\section{Notes and References}

1. P. Gütlich, Y. Garcia and H. A. Goodwin, Chem. Soc. Rev., 2000, 29, 419-427.

2. J. A. Real, A. B. Gaspar and M. C. Munoz, Dalton Trans., 2005, DOI: $10.1039 / \mathrm{b} 501491 \mathrm{c}, 2062-2079$.

3. M. A. Halcrow, Chem. Soc. Rev., 2011, 40, 4119-4142.

4. R. W. Hogue, S. Singh and S. Brooker, Chem. Soc. Rev., 2018, 47, 7303-7338.

5. G. Molnár, M. Mikolasek, K. Ridier, A. Fahs, W. Nicolazzi and

A. Bousseksou, Annalen der Physik, 2019, 0, 1900076.

6. G. Aromí, L. A. Barrios, O. Roubeau and P. Gamez, Coord. Chem. Rev., 2011, 255, 485-546.

7. D. J. Harding, P. Harding and W. Phonsri, Coord. Chem. Rev., 2016, 313, 38-61.

8. S. Hayami, Y. Komatsu, T. Shimizu, H. Kamihata and Y. H. Lee, Coord. Chem. Rev., 2011, 255, 1981-1990.

9. O. Sato, Nat Chem, 2016, 8, 644-656.

10. C. Lefter, V. Davesne, L. Salmon, G. Molnár, P. Demont, A. Rotaru and A. Bousseksou, Magnetochemistry, 2016, 2, 18.

11. K. Senthil Kumar and M. Ruben, Coord. Chem. Rev., 2017, 346, 176-205.

12. G. Molnár, S. Rat, L. Salmon, W. Nicolazzi and A. Bousseksou, Adv. Mater. (Weinheim, Ger.), 2018, 30, 1703862.

13. H. J. Shepherd, I. y. A. Gural'skiy, C. M. Quintero, S. Tricard, L. Salmon, G. Molnár and A. Bousseksou, Nat Commun, 2013, 4. 14. A. Hauser, J. Jeftić, H. Romstedt, R. Hinek and H. Spiering, Coord. Chem. Rev., 1999, 190-192, 471-491. 
15. G. Chastanet, C. Desplanches, C. Baldé, P. Rosa, M. Marchivie and P. Guionneau, Chemistry Squared, 2018, DOI:

https://doi.org/10.28954/2018.csq.07.001, 2.

16. J.-F. Letard, J. Mater. Chem., 2006, 16, 2550-2559.

17. S. Decurtins, P. Gütlich, C. P. Köhler, H. Spiering and A.

Hauser, Chem. Phys. Lett., 1984, 105, 1-4.

18. M. Fumanal, F. Jiménez-Grávalos, J. Ribas-Arino and S. Vela, Inorg. Chem., 2017, 56, 4474-4483.

19. S. Vela, M. Fumanal, J. Ribas-Arino and V. Robert, Phys. Chem. Chem. Phys., 2015, 17, 16306-16314.

20. G. S. Matouzenko, A. Bousseksou, S. Lecocq, P. J. van

Koningsbruggen, M. Perrin, O. Kahn and A. Collet, Inorg. Chem., 1997, 36, 5869-5879.

21. Y. Hasegawa, R. Sakamoto, K. Takahashi and H. Nishihara, Inorg. Chem., 2013, 52, 1658-1665.

22. M. Hostettler, K. W. Törnroos, D. Chernyshov, B. Vangdal and H.-B. Bürgi, Angew. Chem. Int. Ed., 2004, 43, 4589-4594.

23. E. Tailleur, M. Marchivie, P. Negrier, D. Denux, S. Massip, D.

Mondieig, G. Chastanet and P. Guionneau, CrystEngComm, 2019, DOI: $10.1039 / C 9 C E 01137 D$.

24. C. Bartual-Murgui, R. Diego, S. Vela, S. J. Teat, O. Roubeau and G. Aromí, Inorg. Chem., 2018, 57, 11019-11026.

25. M. Weselski, M. Książek, P. Mess, J. Kusz and R. Bronisz, Chem. Commun. (Cambridge, U. K.), 2019, 55, 7033-7036.

26. J. M. Holland, J. A. McAllister, Z. Lu, C. A. Kilner, M.

Thornton-Pett and M. A. Halcrow, Chem. Commun. (Cambridge, $U$. K.), 2001, DOI: 10.1039/b100995h, 577-578

27. S. Vela, J. J. Novoa and J. Ribas-Arino, Phys. Chem. Chem. Phys., 2014, 16, 27012-27024.

28. J. Cirera and E. Ruiz, Comments Inorg. Chem., 2019, 39, 216241.

29. H. Paulsen, V. Schünemann and J. A. Wolny, Eur. J. Inorg. Chem., 2013, 2013, 628-641.

30. O. S. Siig and K. P. Kepp, J. Phys. Chem. A, 2018, 122, 42084217.

31. M. Radoń, Phys. Chem. Chem. Phys., 2019, DOI: 10.1039/C9CP00105K.

32. Q. M. Phung, M. Feldt, J. N. Harvey and K. Pierloot, J. Chem. Theory Comput., 2018, 14, 2446-2455.

33. M. Radoń, K. Gąssowska, J. Szklarzewicz and E. Broclawik, J. Chem. Theory Comput., 2016, 12, 1592-1605.

34. J. Hubbard, Proceedings of the Royal Society of London. Series A. Mathematical and Physical Sciences, 1963, 276, 238-257.

35. M. Shishkin and H. Sato, J. Chem. Phys., 2019, 151, 024102.

36. S. L. Dudarev, G. A. Botton, S. Y. Savrasov, C. J. Humphreys and A. P. Sutton, Phys. Rev. B, 1998, 57, 1505-1509.

37. B. Himmetoglu, A. Floris, S. de Gironcoli and M. Cococcioni, Int. J. Quantum Chem., 2014, 114, 14-49.

38. L. Kabalan, S. F. Matar, M. Zakhour and J. F. Letard, Z. Naturforsch B, 2008, 63, 154

39. S. Lebègue, S. Pillet and J. G. Ángyán, Phys. Rev. B, 2008, 78, 024433.

40. T. Bucko, J. Hafner, S. Lebegue and J. G. Angyan, Phys. Chem. Chem. Phys., 2012, 14, 5389-5396.

41. S. Grimme, J. Comput. Chem., 2006, 27, 1787-1799.

42. S. Vela and H. Paulsen, Inorg. Chem., 2018, 57, 9478-9488.

43. C. Bartual-Murgui, S. Vela, M. Darawsheh, R. Diego, S. J. Teat, O. Roubeau and G. Aromi, Inorganic Chemistry Frontiers, 2017, 4, 1374-1383.

44. C. Bartual-Murgui, S. Vela, O. Roubeau and G. Aromi, Dalton Trans., 2016, 45, 14058-14062.

45. S. Vela and H. Paulsen, Dalton Trans., 2019, 48, 1237-1245. 46. B. Gallois, J. A. Real, C. Hauw and J. Zarembowitch, Inorg. Chem., 1990, 29, 1152-1158.

47. L. Salmon, G. Molnar, S. Cobo, P. Oulie, M. Etienne, T. Mahfoud, P. Demont, A. Eguchi, H. Watanabe, K. Tanaka and A. Bousseksou, New J. Chem., 2009, 33, 1283-1289.

48. C. Krüger, P. Augustín, I. Nemec, Z. Trávníček, H. Oshio, R. Boča and F. Renz, Eur. J. Inorg. Chem., 2013, 2013, 902-915.
49. C. Krüger, P. Augustín, L. u. Dlháň, J. Pavlik, J. Moncol', I. Nemec, R. Boča and F. Renz, Polyhedron, 2015, 87, 194-201. 50. D. J. Harding, W. Phonsri, P. Harding, I. A. Gass, K. S. Murray, B. Moubaraki, J. D. Cashion, L. Liu and S. G. Telfer, Chem. Commun. (Cambridge, U. K.), 2013, 49, 6340-6342. 51. S. Grimme, Chem. Eur, J., 2012, 18, 9955-9964.

52. J. Wu, C. Sousa and C. de Graaf, Magnetochemistry, 2019, 5, 49. 53. L. M. Lawson Daku, Phys. Chem. Chem. Phys., 2018, DOI: 10.1039/C7CP07862E.

54. M. Sorai and S. Seki, J. Phys. Chem. Solids, 1974, 35, 555-570. 55. N. Moliner, M. C. Muñoz, S. Létard, J.-F. Létard, X. Solans, R. Burriel, M. Castro, O. Kahn and J. A. Real, Inorg. Chim. Acta, 1999, 291, 279-288.

56. J. A. Real, M. C. Muñoz, J. Faus and X. Solans, Inorg. Chem., 1997, 36, 3008-3013.

57. M. Sorai, R. Burriel, E. F. Westrum and D. N. Hendrickson, $J$. Phys. Chem. B, 2008, 112, 4344-4350.

58. J.-D. Chai and M. Head-Gordon, Phys. Chem. Chem. Phys., 2008, 10, 6615-6620.

59. E. R. Johnson and A. D. Becke, J. Chem. Phys., 2006, 124, 174104.

60. E. R. Johnson and A. D. Becke, J. Chem. Phys., 2005, 123, 024101.

61. A. D. Becke and E. R. Johnson, J. Chem. Phys., 2005, 123, 154101.

62. S. Grimme, A. Hansen, J. G. Brandenburg and C. Bannwarth, Chem. Rev. (Washington, DC, U. S.), 2016, 116, 5105-5154.

63. S. Grimme, S. Ehrlich and L. Goerigk, J. Comput. Chem., 2011, 32, 1456-1465.

64. M. Cococcioni and S. de Gironcoli, Phys. Rev. B, 2005, 71, 035105 .

65. D. A. Scherlis, M. Cococcioni, P. Sit and N. Marzari, J. Phys. Chem. B, 2007, 111, 7384-7391.

66. D. Vanderbilt, Phys. Rev. B, 1990, 41, 7892-7895.

67. G. Makov and M. C. Payne, Phys. Rev. B, 1995, 51, 4014-4022.

68. Y. Shao, Z. Gan, E. Epifanovsky, A. T. B. Gilbert, M. Wormit, J. Kussmann, A. W. Lange, A. Behn, J. Deng, X. Feng, D. Ghosh, M. Goldey, P. R. Horn, L. D. Jacobson, I. Kaliman, R. Z. Khaliullin, T. Kuś, A. Landau, J. Liu, E. I. Proynov, Y. M. Rhee, R. M. Richard, M. A. Rohrdanz, R. P. Steele, E. J. Sundstrom, H. L. Woodcock, P. M. Zimmerman, D. Zuev, B. Albrecht, E. Alguire, B. Austin, G. J. O. Beran, Y. A. Bernard, E. Berquist, K. Brandhorst, K. B. Bravaya, S. T. Brown, D. Casanova, C.-M. Chang, Y. Chen, S. H. Chien, K. D. Closser, D. L. Crittenden, M. Diedenhofen, R. A. DiStasio, H. Do, A. D. Dutoi, R. G. Edgar, S. Fatehi, L. Fusti-Molnar, A. Ghysels, A. Golubeva-Zadorozhnaya, J. Gomes, M. W. D. HansonHeine, P. H. P. Harbach, A. W. Hauser, E. G. Hohenstein, Z. C. Holden, T.-C. Jagau, H. Ji, B. Kaduk, K. Khistyaev, J. Kim, J. Kim, R. A. King, P. Klunzinger, D. Kosenkov, T. Kowalczyk, C. M. Krauter, K. U. Lao, A. D. Laurent, K. V. Lawler, S. V. Levchenko, C. Y. Lin, F. Liu, E. Livshits, R. C. Lochan, A. Luenser, P. Manohar, S. F. Manzer, S.-P. Mao, N. Mardirossian, A. V. Marenich, S. A. Maurer, N. J. Mayhall, E. Neuscamman, C. M. Oana, R. OlivaresAmaya, D. P. O'Neill, J. A. Parkhill, T. M. Perrine, R. Peverati, A. Prociuk, D. R. Rehn, E. Rosta, N. J. Russ, S. M. Sharada, S. Sharma, D. W. Small, A. Sodt, T. Stein, D. Stück, Y.-C. Su, A. J. W. Thom, T. Tsuchimochi, V. Vanovschi, L. Vogt, O. Vydrov, T. Wang, M. A. Watson, J. Wenzel, A. White, C. F. Williams, J. Yang, S. Yeganeh, S. R. Yost, Z.-Q. You, I. Y. Zhang, X. Zhang, Y. Zhao, B. R. Brooks, G. K. L. Chan, D. M. Chipman, C. J. Cramer, W. A. Goddard, M. S. Gordon, W. J. Hehre, A. Klamt, H. F. Schaefer, M. W. Schmidt, C. D. Sherrill, D. G. Truhlar, A. Warshel, X. Xu, A. Aspuru-Guzik, R. Baer, A. T. Bell, N. A. Besley, J.-D. Chai, A. Dreuw, B. D. Dunietz, T. R. Furlani, S. R. Gwaltney, C.-P. Hsu, Y. Jung, J. Kong, D. S. Lambrecht, W. Liang, C. Ochsenfeld, V. A. Rassolov, L. V. Slipchenko, J. E. Subotnik, T. Van Voorhis, J. M. Herbert, A. I. Krylov, P. M. W. Gill and M. Head-Gordon, Mol. Phys., 2015, 113, 184-215.

69. F. Weigend, Phys. Chem. Chem. Phys., 2006, 8, 1057-1065. 
70. F. Weigend and R. Ahlrichs, Phys. Chem. Chem. Phys., 2005, 7, 3297-3305.

71. S. Grimme, J. Antony, S. Ehrlich and H. Krieg, J. Chem. Phys., 2010, 132, 154104.

72. S. Grimme and M. Steinmetz, Phys. Chem. Chem. Phys., 2013, 15, 16031-16042.

73. J. Moellmann and S. Grimme, J. Phys. Chem. C, 2014, 118,

7615-7621.

74. M. Reiher, Inorg. Chem., 2002, 41, 6928-6935.

75. M. Reiher, O. Salomon and B. Artur Hess, Theor. Chem. Acc., 2001, 107, 48-55.

76. M. Güell, M. Solà and M. Swart, Polyhedron, 2010, 29, 84-93.

77. M. Swart, J. Chem. Theory Comput., 2008, 4, 2057-2066.

78. J. Cirera, M. Via-Nadal and E. Ruiz, Inorg. Chem., 2018, DOI: 10.1021/acs.inorgchem.8b01821.

79. K. S. Murray, W. Phonsri, G. N. L. Jameson and B. Lewis,

Chem. Commun. (Cambridge, U. K.), 2019, DOI:

10.1039/C9CC07416C. 